\title{
CASE OF DERMOID SARCOMA OF RECTUS ABDOMINALIS.
} By HAROLD DODD, Ch.M., F.R.C.S.

(Surgeon, King George Hospital, Ilford, and Royal Hospital, Richmond.)

30TH OCTOBER, I93I. Mrs. G. D., aged 28, when first seen was complaining of a lump in the abdomen which she noticed casually some time after lifting a weight. The swelling was immediately above and to the left of the umbilicus in the substance of the left rectus muscle. It measured I inch in circumference, was hard and round but not tender.

The provisional diagnosis of a dermoid tumour in the rectus muscle was made.

IOTH NOVEMBER, I93I. Operation. The tumour was widely excised with the overlying subcutaneous tissue, rectus sheath and surrounding rectus muscle. The tumour was slightly adherent to the posterior rectus sheath and a small circle of it with peritoneum was included in the excised tissue; this gap was easily sutured. The deficiency in the rectus muscle was closed by turning over a flap of anterior rectus sheath from the corresponding segment at the opposite side as shown by diagram I. The patient made a good recovery and experienced no discomfort.

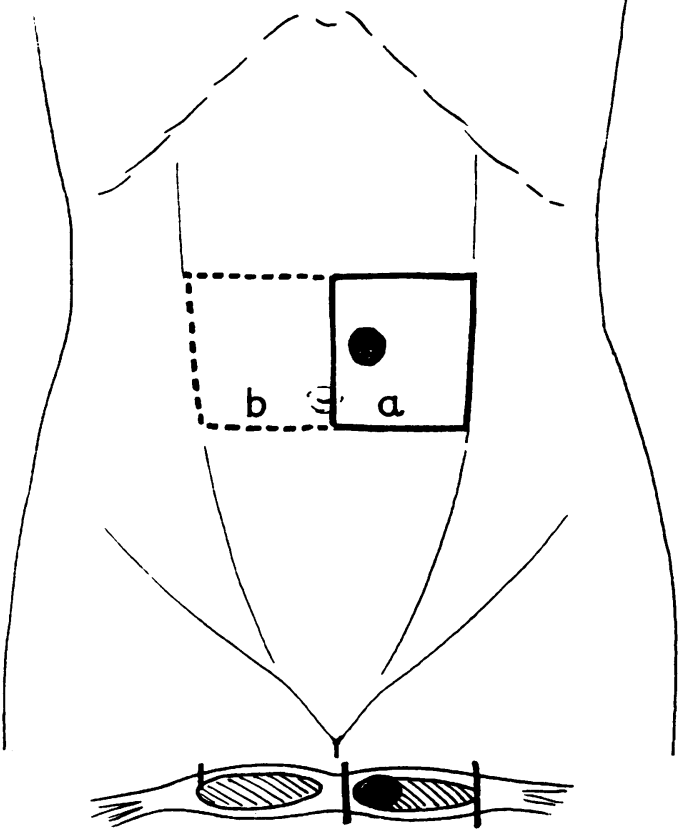

Fig. I.-(a) The area excised.

(b) The flap of rectus sheath reflected.

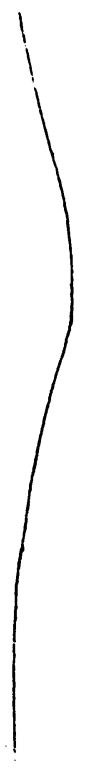

Fig. 2.-The flap (b) turned over and sutured to close the deficiency in rectus.

27TH November, I93I. Microscopical section showed the tumour to be a fibrosarcoma infiltrating muscle.

25TH November, I932. The patient stated she felt quite well and was able to do all her duties but she thought she could feel another lump the size of a small walnut in the scar at the mid-line. This was confirmed on clinical examination.

23RD JANUARY, I933. She was readmitted to hospital for a second operation. A transverse incision was made across the abdomen and the wound edges were reflected above and below the swelling which appeared to be involving the mid-line and also the central part of the right rectus. The entire area was excised en bloc. It included 


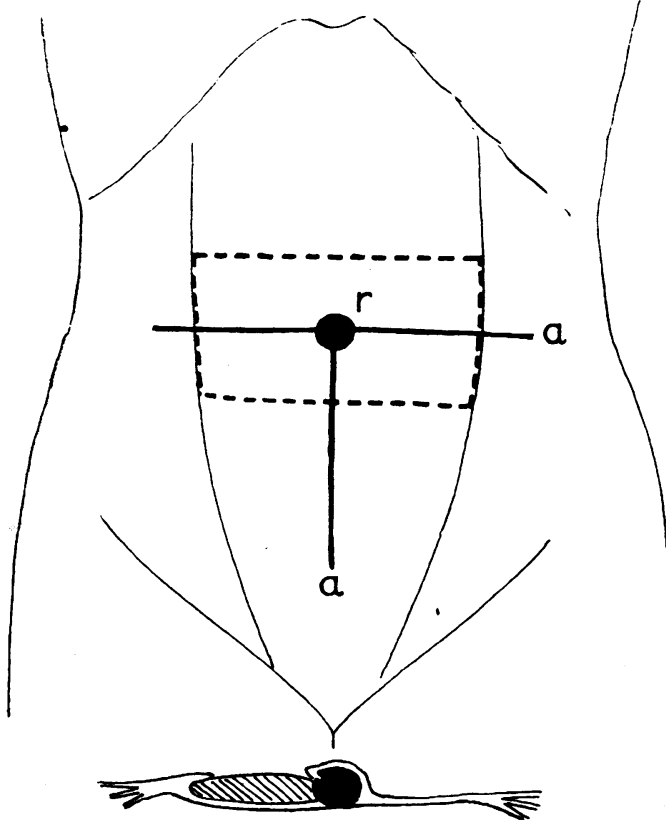

Fig. 3.-lllustrates (a) The T-shaped incision centred about the recurrence $(r)$.

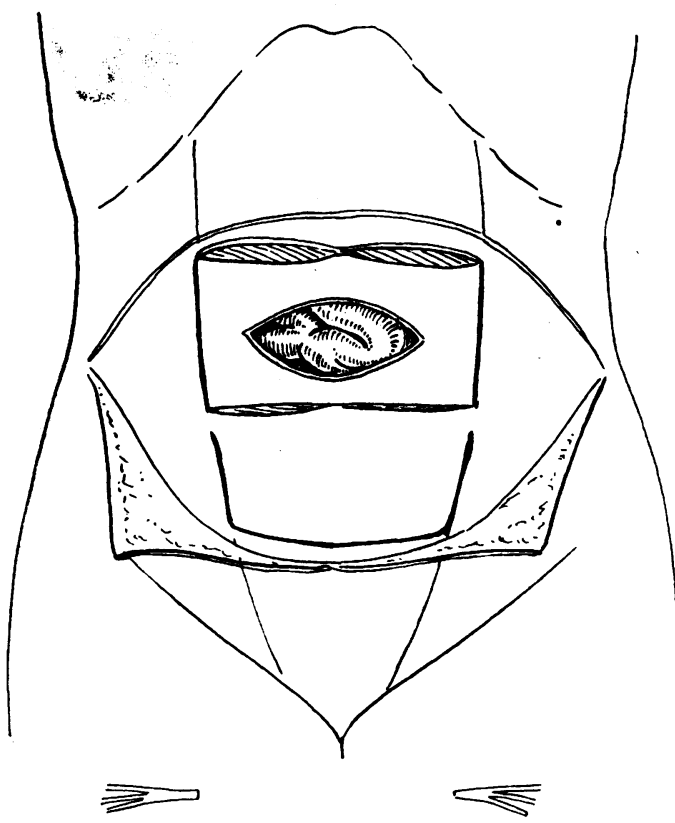

Fig. 4.- - Illustrates the deficiency in the abdominal wall from excision of a section of right and left rectus and also the underlying peritoneum. The firm line below outlines a flap of rectus sheath that was reflected and turned back to close this gap. (a) the flap of rectus muscle turned over from the opposite side at the previous operation; (b) the underlying peritoneum; (c) the affected segment of the right rectus muscle which contained the tumour; and (d) the posterior rectus sheath and peritoneum. There was now a deficiency in the abdominal wall consisting of a segment of the right and left rectus muscles with the associated anterior and posterior sheath. The gap in the posterior rectus sheath was closed by raising the pelvis and head and shoulders on the table and mobilizing the peritoneum and rectus sheath. The breach in the muscle was repaired by extending the incision downwards in the mid-line and reflecting from both the right and left rectus muscles a large flap of anterior rectus sheath. This was turned up and sutured to the external rectus laterally and to rectus sheath transversely. The patient made a good recovery. See diagram, Figs. $3,4 \& 5$.

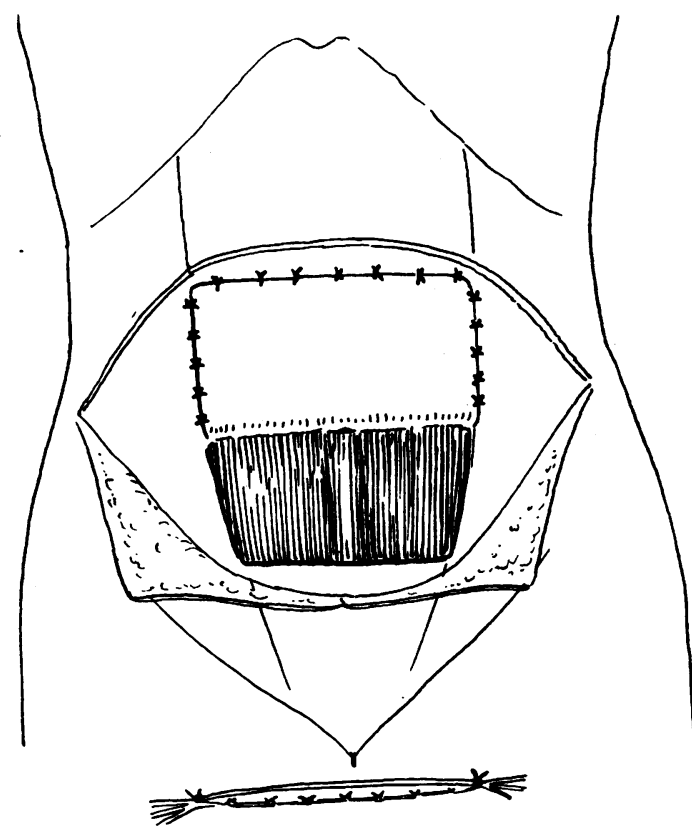

Fig. 5.-Illustrates the deficiency in the abdominal wall closed by the up-turned flap of rectus sheath. 
2ND FEBRUARY, I933. Microscopical section showed the tumour to be a fibrosarcoma (dermoid of rectus muscle).

Radium was applied on Columbia paste with a view to inhibiting further growth.

The patient has been seen regularly since, at first three monthly, later twice a year, and now annually.

I4TH JANUARY, I938. When last interviewed there was no sign of recurrence and she is living the life of an active house-wife. She has never experienced inconvenience or signs of weakness. The photograph B illustrates the contour of her

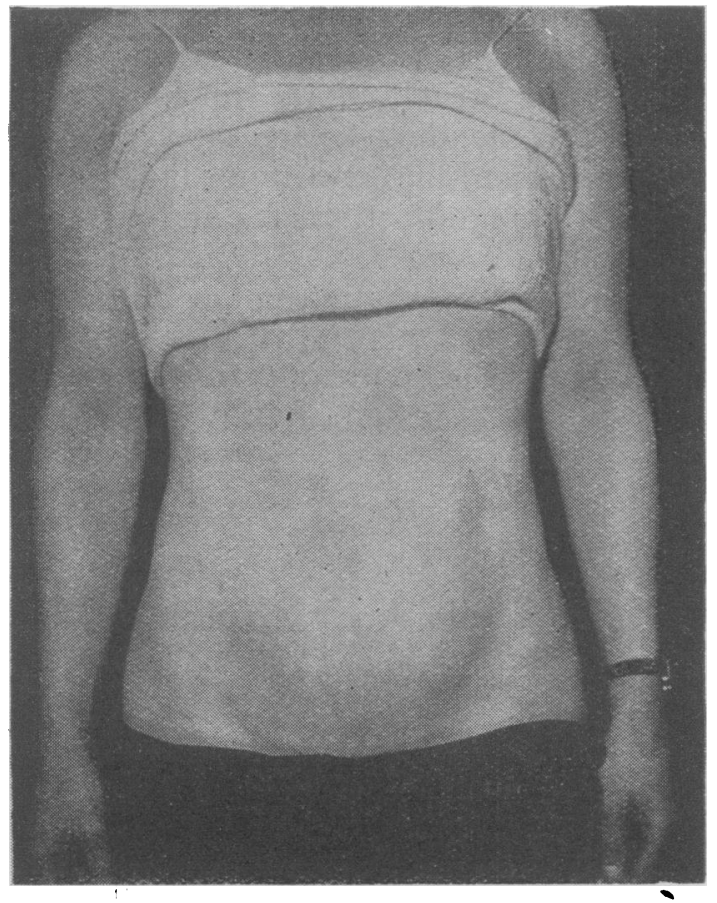

Fig. A. Shows the abdomen six years after operation.

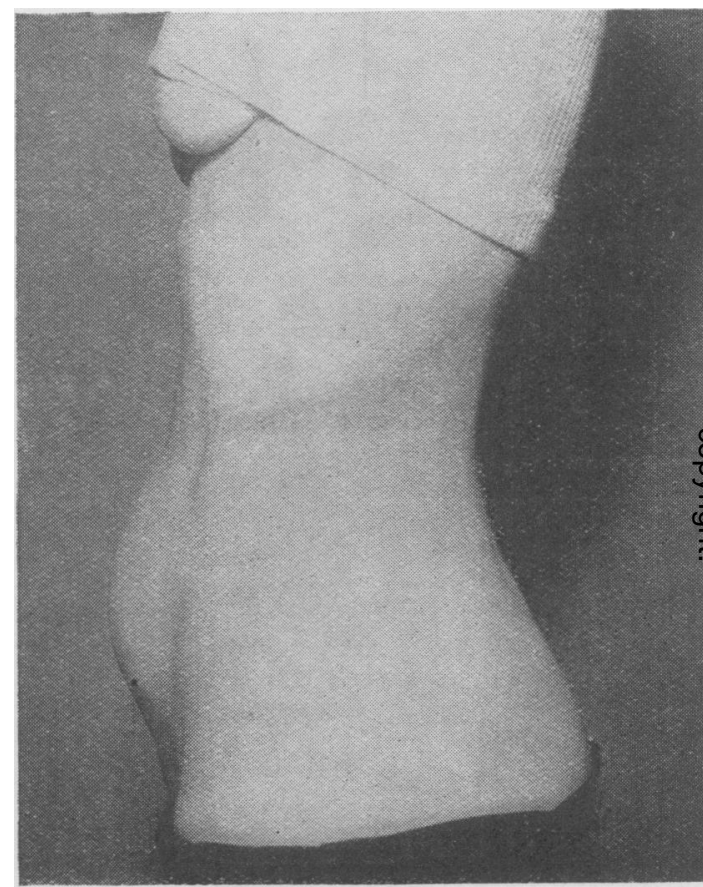

Fig. B. Side view of the abdomen six years later.

abdomen seven years after the last operation; there is no sign of a hernia. Successive house surgeons have examined her and weakness or bulging of the abdominal wall has never been commented on. If such a case confronted me again, I think I should turn the flap down from the upper recti rather than from below, although a better result could hardly be desired.

This case is reported to illustrate that

(a) The widest possible heroic excision of a dermoid sarcoma is necessary.

(b) So far as the abdominal wall is concerned a gross defect can be satisfactorily repaired so as to permit a normal, active life.

(c) Prophylactic radium is advisable in cases of dermoid sarcoma.

(d) The prognosis is a fair one. 